\title{
Critical collapse and the primordial black hole initial mass function
}

\author{
Anne M. Green \\ Astronomy Centre, University of Sussex, Brighton BN1 9QJ, U. K. \\ and \\ Astronomy Unit, School of Mathematical Sciences, Queen Mary and Westfield College, \\ Mile End Road, London, E1 4NS, U. K. (present address) \\ Andrew R. Liddle \\ Astronomy Centre, University of Sussex, Brighton BN1 9QJ, U. K. \\ and \\ Astrophysics Group, The Blackett Laboratory, Imperial College, London, SW7 2BZ, U. K. (present address)
}

(June 11, 2018)

\begin{abstract}
It has normally been assumed that primordial black holes (PBHs) always form with mass approximately equal to the mass contained within the horizon at that time. Recent work studying the application of critical phenomena in gravitational collapse to $\mathrm{PBH}$ formation has shown that in fact, at a fixed time, PBHs with a range of masses are formed. When calculating the $\mathrm{PBH}$ initial mass function it is usually assumed that all PBHs form at the same horizon mass. It is not clear, however, that it is consistent to consider the spread in the mass of PBHs formed at a single horizon mass, whilst neglecting the range of horizon masses at which PBHs can form. We use the excursion set formalism to compute the PBH initial mass function, allowing for PBH formation at a range of horizon masses, for two forms of the density perturbation spectrum. First we examine power-law spectra with $n>1$, where PBHs form on small scales. We find that, in the limit where the number of PBHs formed is small enough to satisfy the observational constraints on their initial abundance, the mass function approaches that found by Niemeyer and Jedamzik under the assumption that all PBHs form at a single horizon mass. Second, we consider a flat perturbation spectrum with a spike at a scale corresponding to horizon mass $\sim 0.5 M_{\odot}$, and compare the resulting $\mathrm{PBH}$ mass function with that of the MACHOs (MAssive Compact Halo Objects) detected by microlensing observations. The predicted mass spectrum appears significantly wider than the steeply-falling spectrum found observationally.
\end{abstract}

PACS numbers: 98.80.-k

astro-ph/9901268

\section{INTRODUCTION}

There has been a lot of interest recently in numerical studies of gravitational collapse, carried out for a wide range of matter models, which appear to exhibit critical phenomena: self-similarity, universality and power-law scaling of the black hole mass (for a review and extensive references see e.g. [1]). Consider a smooth one-parameter family of initial data described by a parameter $p$, such that for $p>p_{\mathrm{c}}$ a black hole is formed and for $p<p_{\mathrm{c}}$ no black hole is formed. Numerical simulations (originally carried out by Choptuik for the case of a massless scalar field [2] and by Evans and Coleman [3] for a perfect fluid with equation of state $p=\rho / 3$ ) show that the black hole mass, $M_{\mathrm{BH}}$, scales as

$$
M_{\mathrm{BH}} \propto\left(p-p_{\mathrm{c}}\right)^{\gamma},
$$

for $p \simeq p_{\mathrm{c}}$, where $\gamma$ is a universal scaling exponent which is independent, for a given matter model, of the choice of $p$ and the initial shape of the density fluctuations.

It has been pointed out by Niemeyer and Jedamzik [4] that this work has an astrophysical application to primordial black hole $(\mathrm{PBH})$ formation. For critical phenomena to occur, the initial distribution of fluctuations must be such that most of the collapsing fluctuations have magnitude not too far above the critical magnitude for collapse. This situation arises in the formation of PBHs from density perturbations in the early universe, since the distribution of large PBH forming fluctuations falls off rapidly, roughly as $P(\delta) \propto \exp \left(-\delta^{2}\right)$ where $\delta=\delta \rho / \rho$. Generally this is not the case during astrophysical black hole formation, such as stellar collapse where mass scales such as the Chandrasekhar mass are introduced due to degeneracy pressure and other effects violating the scale-free behaviour. .

Niemeyer and Jedamzik [0] have investigated the evolution of spherically-symmetric perturbations of the energy density in unperturbed Hubble flow during radia-

\footnotetext{
* Such physical effects may also occur on small scales in the case of PBH formation so that arbitrarily small mass PBHs will not be formed, but this does not affect the general validity of Eq. (囵).
} 
tion domination for three different perturbation shapes at horizon crossing: a gaussian overdensity, which tends to the background Friedmann-Robertson-Walker metric at infinity, a Mexican Hat function, and an unspecified fourth-order polynomial. In the latter two cases the inner overdensity is compensated by a surrounding underdense region. The initial conditions are tuned to supercriticality by adjusting the amplitude of the perturbations. Their simulations confirm the existence of the mass scaling relation in the case of $\mathrm{PBH}$ formation:

$$
M_{\mathrm{BH}}=k M_{\mathrm{H}}\left(\delta-\delta_{\mathrm{c}}\right)^{\gamma},
$$

where $M_{\mathrm{H}}$ is the horizon mass at the time the fluctuation enters the horizon, $\delta$ is defined as the additional mass inside the horizon radius in units of the horizon mass and $\gamma \approx 0.37$ for all three shapes of perturbation. For gaussian shaped fluctuations $k=11.9$ and $\delta_{\mathrm{c}}=0.70$, for the Mexican Hat fluctuations $k=2.85$ and $\delta_{\mathrm{c}}=0.67$, and for the fourth-order polynomial $k=2.39$ and $\delta_{\mathrm{c}}=0.71$, suggesting that $\delta_{\mathrm{c}} \sim 0.7$ for all perturbation shapes. This value of $\delta_{\mathrm{c}}$ is about a factor of 2 larger than the value found analytically [6] by requiring that the over-dense region exceeds its Jeans length at the time that it stops expanding.

The formation, at a fixed horizon mass, of PBHs with a range of masses is a significant change from the standard picture where it is assumed that all $\mathrm{PBHs}$ are formed with $M_{\mathrm{BH}} \approx M_{\mathrm{H}}$ [7,6]. Niemeyer and Jedamzik have determined the PBH initial mass function under the assumption that all PBHs form at the same horizon mass. This assumption is often used; for example, with a powerlaw spectrum with spectral index greater than one it is assumed that the vast majority of the black hole formation occurs at the shortest possible scale. The usual justification is that PBH formation is extremely sensitive to the amplitude of the perturbations, and hence inherits a strong dependence on scale even if the variation of the spectrum is weak. It is not clear, however, that it is consistent to consider the spread in the mass of PBHs formed at a single horizon mass, whilst neglecting the range of horizon masses at which the PBHs are formed. Investigation of this issue is the main purpose of this paper. In the end, we shall in fact find that this assumption works very well in the cases of astrophysical interest.

Heuristically, to find the mass of a $\mathrm{PBH}$ formed at a given point in space we need to smooth the density field at that point, $\delta(\mathbf{x})$, on a range of mass scales, to produce $\delta(M)$, where $M$ is the smoothing scale, and then evaluate each $\delta(M)$ at the time when that scale crosses the horizon, $M=M_{\mathrm{H}}$. If $\delta\left(M=M_{\mathrm{H}}\right)>\delta_{\mathrm{c}}$ then a PBH is

\footnotetext{
${ }^{\dagger}$ Realistic PBH formation differs from the study of perfect fluid collapse in Ref. 3, where the initial data was embedded in an asymptotically flat space-time, in that the background spacetime is Friedmann-Robertson-Walker.
}

formed, with mass given by Eq. (2), at that horizon mass. However, that condition will be satisfied for a range of smoothing masses; the actual mass of the PBH formed at the point $\mathrm{x}$ is given by the largest value of $M_{\mathrm{BH}}$ found as the smoothing scale is decreased, which will not be the largest smoothing scale giving a density contrast above the threshold due to the dependence of the mass on $(\delta-$ $\delta_{\mathrm{c}}$ ). To find the optimal smoothing mass, we use the excursion set formalism [8] which was introduced in largescale structure studies to determine the mass function, merger rates and clustering of collapsed objects e.g. [8,9].

\section{THE EXCURSION SET FORMALISM}

\section{A. Standard formalism}

To examine the density perturbations on a given scale we must smooth the density field, as described above, using a window function $W\left(R_{\mathrm{f}},\left|\mathbf{x}-\mathbf{x}^{\prime}\right|\right)$ with radius $R_{\mathrm{f}}$. The density contrast is defined as $\delta(\mathbf{x})=(\rho(\mathbf{x})-\bar{\rho}) / \bar{\rho}$, and the smoothed version is given by:

$$
\begin{aligned}
\delta\left(R_{\mathrm{f}}, \mathbf{x}\right) & =\int_{0}^{\infty} W\left(R_{\mathrm{f}},\left|\mathbf{x}-\mathbf{x}^{\prime}\right|\right) \delta\left(\mathbf{x}^{\prime}\right) \mathrm{d}^{3} \mathbf{x}^{\prime} \\
& =\frac{1}{(2 \pi)^{3}} \int_{0}^{\infty} W\left(k R_{\mathrm{f}}\right) \delta(\mathbf{k}) \exp (-i \mathbf{k} \cdot \mathbf{x}) \mathrm{d}^{3} \mathbf{k},
\end{aligned}
$$

where $W\left(k R_{\mathrm{f}}\right)$ and $\delta(\mathbf{k})$ are the Fourier transform of the window function and the unsmoothed density contrast respectively, with $k=|\mathbf{k}|$. The variance of the mass distribution $\sigma^{2}\left(R_{\mathrm{f}}\right)$, as defined in [10], is given by

$$
\sigma^{2}\left(R_{\mathrm{f}}\right)=\int_{0}^{\infty} W\left(k R_{\mathrm{f}}\right) \mathcal{P}_{\delta}(k) \frac{\mathrm{d} k}{k},
$$

where $\mathcal{P}_{\delta}(k)$ is the spectrum of $\delta$

$$
\mathcal{P}_{\delta}(k)=\frac{k^{3}}{2 \pi^{2}}\left\langle|\delta(\mathbf{k})|^{2}\right\rangle .
$$

The effect of varying $R_{\mathrm{f}}$, at fixed time, can be found by differentiating Eq. (3) [9]:

$$
\begin{aligned}
\frac{\partial \delta\left(R_{\mathrm{f}}, \mathbf{x}\right)}{\partial R_{\mathrm{f}}}= & \frac{1}{(2 \pi)^{3}} \int_{0}^{\infty} \delta(\mathbf{k}) \frac{\partial W\left(k R_{\mathrm{f}}\right)}{\partial R_{\mathrm{f}}} \\
& \times \exp (-i \mathbf{k} \cdot \mathbf{x}) \mathrm{d} \mathbf{k} \equiv \eta\left(R_{\mathrm{f}}, \mathbf{x}\right) .
\end{aligned}
$$

This has the form of a Langevin equation; the change in $\delta\left(R_{\mathrm{f}}, \mathbf{x}\right)$ when $R_{\mathrm{f}}$ is changed is given in terms of a stochastic force $\eta\left(R_{\mathrm{f}}, \mathbf{x}\right)$, which depends on the form of the window function used.

A particularly good choice of window function if one wants to make analytical progress is the sharp $k$-space window function

$$
\tilde{W}\left(k R_{\mathrm{f}}\right)=\Theta\left(1-k R_{\mathrm{f}}\right) .
$$


Its strength is that the only effect of decreasing the smoothing radius is to add new Fourier modes of the unsmoothed density contrast $\delta(\mathbf{x})$ to the integral for $\sigma^{2}$. For gaussian perturbations, as we will be assuming throughout, fit these new modes are uncorrelated with the ones already included in the integral on a larger smoothing scale. The change in $\delta\left(R_{\mathrm{f}}, \mathbf{x}\right)$ caused by the new modes is therefore independent of its value on the larger smoothing scale, leading to a random walk without memory. For other choices of window function, including the commonly-used top-hat and gaussian forms, this nice property doesn't hold since changing $R_{f}$ alters the contribution to $\sigma^{2}$ from modes of all wavenumbers. That said, although the sharp $k$-space window function is calculationally advantageous, one wouldn't expect physical results to be all that dependent on the choice of smoothing.

With the sharp $k$-space window function, Eq. (6) simplifies to

$$
\frac{\partial \delta(\mathbf{x}, \Lambda)}{\partial \Lambda}=\zeta(\Lambda)
$$

independent of position, where $\Lambda \equiv \sigma^{2}\left(R_{\mathrm{f}}\right)$ is a pseudotime variable and

$$
\left\langle\zeta\left(\Lambda_{1}\right) \zeta\left(\Lambda_{2}\right)\right\rangle=\delta_{\mathrm{D}}\left(\Lambda_{1}-\Lambda_{2}\right),
$$

the right-hand side being the Dirac delta function.

Eq. (8) can be integrated to give

$$
\delta(\Lambda+\gamma)-\delta(\Lambda)=\sqrt{\gamma} G
$$

where $G$ is a gaussian random variable with mean zero and unit variance, and since this equation is exact the step size, $\gamma$, can be chosen freely. Stochastic processes which are governed by this equation, such as self diffusion in a hard sphere gas, are known as Wiener processes. We can think of the values of $\delta(\Lambda)$ produced as $\Lambda$ is increased (or equivalently $R_{\mathrm{f}}$ decreased) as mapping out a trajectory, analogous to the path of a self-diffusing particle. Each trajectory has the initial condition $\delta(0)=0$ since in the limit that $R_{\mathrm{f}} \rightarrow \infty, \delta\left(R_{\mathrm{f}}, \mathbf{x}\right) \rightarrow 0$ by definition of the mean density.

Using Eq. (10) and a random number generator, we can generate an ensemble of trajectories each representing the variation, with smoothing scale, of the smoothed density field at a different point in space. The trajectories of $\delta(\Lambda)$ produced for a chosen range of $\Lambda$ values are independent of the form of the power spectrum. To relate each value of $\Lambda$ to a mass scale, $M$, we need to choose a form for the power spectrum and then use Eq. (4) to find the relationship between $\Lambda$ and $M$.

\footnotetext{
$\ddagger$ The assumption of gaussianity has been challenged for large PBH-forming fluctuations 11; however it is reasonable to maintain this assumption in order to assess the effect of critical collapse on the PBH mass function.
}

\section{B. Application to PBH formation}

The trajectories give the variation of $\delta(M)$ at fixed time; however, the condition for $\mathrm{PBH}$ formation on a given scale, $\delta(M)>\delta_{\mathrm{c}}$, and Eq. (2) which gives the mass of the PBH produced, apply when that scale crosses the horizon, i.e. $M=M_{\mathrm{H}}$. We must therefore choose a fixed time at which to generate our trajectories, and then evolve each value of $\delta(M)$ forwards, or backwards, in time to the epoch at which that scale crosses the horizon. During radiation domination perturbations which are outside the horizon grow as 10

$$
\delta \propto t \propto M_{\mathrm{H}}
$$

and therefore

$$
\delta_{\mathrm{hc}}\left(M_{\mathrm{H}}\right)=\delta_{\mathrm{ft}}\left(M_{\mathrm{H}}\right)\left(\frac{M_{\mathrm{H}}}{M_{\mathrm{H}, \min }}\right),
$$

where 'hc' and 'ft' denote quantities evaluated at horizon crossing and the chosen fixed time respectively. For $\mathrm{PBH}$ formation we require $\delta_{\mathrm{ft}}\left(M_{\mathrm{H}}\right)>\delta_{\mathrm{c}, \mathrm{ft}}\left(M_{\mathrm{H}}\right)$ where

$$
\delta_{\mathrm{c}, \mathrm{ft}}\left(M_{\mathrm{H}}\right)=\delta_{\mathrm{c}}\left(\frac{M_{\mathrm{H}}}{M_{\mathrm{H}, \min }}\right) \text {. }
$$

\section{POWER-LAW POWER SPECTRA}

We first examine the case where the primordial power spectrum is a power-law

$$
\mathcal{P}(\delta) \propto k^{n+3},
$$

[equivalently $P(k) \propto k^{n}$, where $P(k)=\left\langle\left|\delta_{\mathbf{k}}\right|^{2}\right\rangle$ and $n$ is known as the spectral index]. Inserting this form for the power spectrum into Eq. (4) gives $\sigma^{2}(R) \propto R^{-(n+3)}$.

During radiation domination the mass in a comoving region evolves, reducing as $1 / a$. Ultimately we are interested in the mass associated with a given scale when that scale crosses the horizon; horizon crossing is given by $R \propto 1 / a H \propto t^{1 / 2} \propto M_{\mathrm{H}}^{1 / 2}$ 12 so that, still at fixed time,

$$
\sigma^{2}\left(M_{\mathrm{H}}\right)=\sigma^{2}\left(M_{\mathrm{H}, \min }\right)\left(\frac{M_{\mathrm{H}}}{M_{\mathrm{H}, \min }}\right)^{-(n+3) / 2} .
$$

where we have chosen our fixed time as the epoch immediately after the reheating period at the end of inflation when the horizon mass has its minimum value, $M_{\mathrm{H}, \mathrm{min}}$, which is determined by the reheat temperature, $T_{\mathrm{RH}}$, via

$$
M_{\mathrm{H}, \min }=M_{\mathrm{H}, 0}\left(\frac{T_{\mathrm{eq}}}{T_{\mathrm{RH}}}\right)^{2}\left(\frac{T_{0}}{T_{\mathrm{eq}}}\right)^{3 / 2} .
$$

We can obtain $\sigma\left(M_{\mathrm{H}, \min }\right)$ from the mass variance on the present horizon scale, using the variation with $M$ 
of the mass variance at horizon crossing (for details see Ref. [12]):

$\sigma\left(M_{\mathrm{H}, \min }\right)=\sigma\left(M_{\mathrm{H}, 0}\right)\left(\frac{M_{\mathrm{eq}}}{M_{0}}\right)^{(1-n) / 6}\left(\frac{M_{\mathrm{H}, \min }}{M_{\mathrm{eq}}}\right)^{(1-n) / 4}$

where ' 0 ' and 'eq' denote quantities evaluated at the present day and matter-radiation equality, and $\sigma\left(M_{\mathrm{H}, 0}\right)=9.5 \times 10^{-5}$ using the COBE normalization $[12]$.

There are a number of well-known constraints on the abundance of PBHs over a wide range of mass scales; for an up-to-date review see Ref. [13]. Typically only of order $10^{-20}$ of the energy density of the universe, at the time that they form, can go into PBHs. This leads to the constraint that $n$ must be less than about 1.25 [12], with some dependence on the value of $M_{\mathrm{H} \text {,min }}$. It is obviously not feasible to run simulations where only one trajectory in $10^{20}$ produces a $\mathrm{PBH}$. Using larger values of $n$, around 1.3, a manageable number of trajectories will form $\mathrm{PBHs}$ but the values of $\delta_{\mathrm{hc}}\left(M_{\mathrm{H}}\right)$, and hence $M_{\mathrm{BH}}$ produced will typically be larger than when $n \sim 1.25$. We can however examine how the distribution of $\mathrm{PBH}$ masses behaves as $n$ is decreased and compare its limiting behaviour with the mass function found analytically by Niemeyer and Jedamzik under the assumption that all PBHs form at the same horizon mass [4]

$$
\begin{aligned}
\frac{\mathrm{d} N}{\mathrm{~d}\left(\ln M_{\mathrm{BH}}\right)}= & \frac{1}{\sqrt{2 \pi} \sigma \gamma}\left(\frac{M_{\mathrm{BH}}}{k M_{\mathrm{H}}}\right)^{1 / \gamma} \\
& \times \exp \left(-\frac{\left(\delta_{\mathrm{c}}+\left(M_{\mathrm{BH}} / k M_{\mathrm{H}}\right)^{1 / \gamma}\right)^{2}}{2 \sigma^{2}}\right),
\end{aligned}
$$

where $\mathrm{d} N$ is the number of PBHs per logarithmic mass interval $\mathrm{d}\left(\ln M_{\mathrm{BH}}\right)$, and $\sigma \equiv \sigma\left(M_{\mathrm{H}}\right)$. The Niemeyer and Jedamzik mass function (NJMF) will be valid if the distribution of the horizon masses at which the PBHs are formed is close to a delta function.

As an example we choose $M_{\mathrm{H}, \mathrm{min}}=10^{10} \mathrm{~g}$, which corresponds to $T_{\mathrm{RH}} \approx 3 \times 10^{11} \mathrm{GeV}$, and in Eq. (2) we use the values of $k$ and $\delta_{\mathrm{c}}$ from Ref. [5] for Mexican hat shaped fluctuations. Our general conclusions will however be independent of these choices. Since the probability of $\mathrm{PBH}$ formation falls off rapidly with increasing horizon mass we generate trajectories using 1000 equally spaced steps in $\Lambda$, giving adequate coverage of the range of horizon masses at which PBHs form. Fig. 1 shows a typical PBH forming trajectory with $n=1.3$.

We ran simulations producing $1000 \mathrm{PBHs}$ for each of $n=1.310,1.305,1.300$, and 1.295 and in each case found the distributions (smoothed with a gaussian to remove the effects of discreteness from the finite number of PBHs) of the PBHs masses and the horizon masses at which they formed. We denote these as $\chi\left(M_{\mathrm{BH}}\right)$ and $\chi\left(M_{\mathrm{H}}\right)$ respectively, so that the number of $\mathrm{PBHs}$

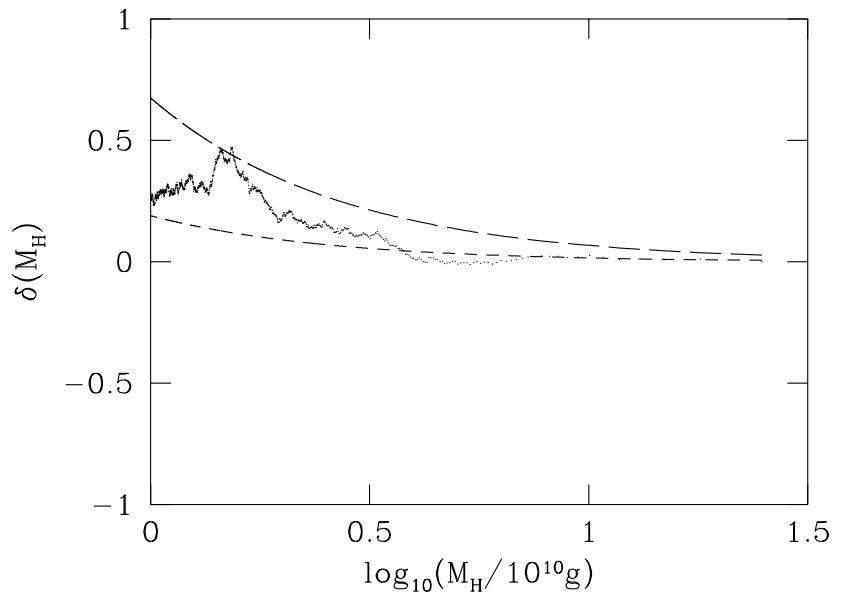

FIG. 1. A typical PBH forming trajectory resulting from a power-law spectrum with $n=1.3$. The dotted points are the values of $\delta\left(M_{\mathrm{H}}\right)$, the long-dashed line shows the threshold, at the fixed time for $\mathrm{PBH}$ formation $\delta_{\mathrm{c}, \mathrm{ft}}\left(M_{\mathrm{H}}\right)$, and the short-dashed line shows the typical size of perturbations on each scale, $\sigma\left(M_{\mathrm{H}}\right)$.

per logarithmic mass interval produced in our simulations is given by $\chi\left(M_{\mathrm{BH}}\right) \mathrm{d}(\ln M)$. The upper panel of Fig. 2 shows the smoothed mass distributions $\chi\left(M_{\mathrm{BH}}\right)$, along with the Niemeyer and Jedamzik mass function (smoothed on the same scale to allow direct comparison) evaluated for $M_{\mathrm{H}}=10^{10} \mathrm{~g}$ and $\sigma=0.032$, the value of $\sigma\left(10^{10} \mathrm{~g}\right.$ ) for $n=1.23$ (the maximum value of $n$ allowed for $M_{\mathrm{H}, \min }=10^{10} \mathrm{~g}$ by the observational constraints). We normalize all mass distributions to 1 at their peak to facilitate comparison. As $n$ is decreased, the $\mathrm{PBH}$ mass function tends towards that found by assuming that all $\mathrm{PBH}$ form at a single horizon mass. We can test this further by examining the horizon masses at which the PBHs form, rather than their actual masses. The lower panel shows the smoothed distributions of the horizon masses at which the PBHs formed, plotted along with a delta function centered on $M_{\mathrm{H}}=10^{10} \mathrm{~g}$ and smoothed on the same scale. As $n$ is decreased the horizon mass distribution narrows.

We therefore conclude that for power-law spectra, with slope satisfying the constraints from the observational limits on $\mathrm{PBH}$ abundance, the assumption that all $\mathrm{PBH}$ form at the same horizon mass is a good approximation. Yokoyama 14] has re-evaluated the constraints on the initial mass fraction of PBHs, taking account of the mass scaling relation under this assumption. He found that the constraints on the initial abundance of PBHs are unchanged if formation occurs at $M_{\mathrm{H}}<5 \times 10^{14} \mathrm{~g}$. The limit on the abundance of PBHs with $M_{\mathrm{BH}}=5 \times 10^{14} \mathrm{~g}$, which are evaporating today, coming from the $\gamma$-ray background, now leads to stronger limits on the initial abundance if $5 \times 10^{14} \mathrm{~g}<M_{\mathrm{H}}<10^{17} \mathrm{~g}$, as for horizon masses in this range the low-mass tail of the distribution has a significant population light enough to be evaporating today. 

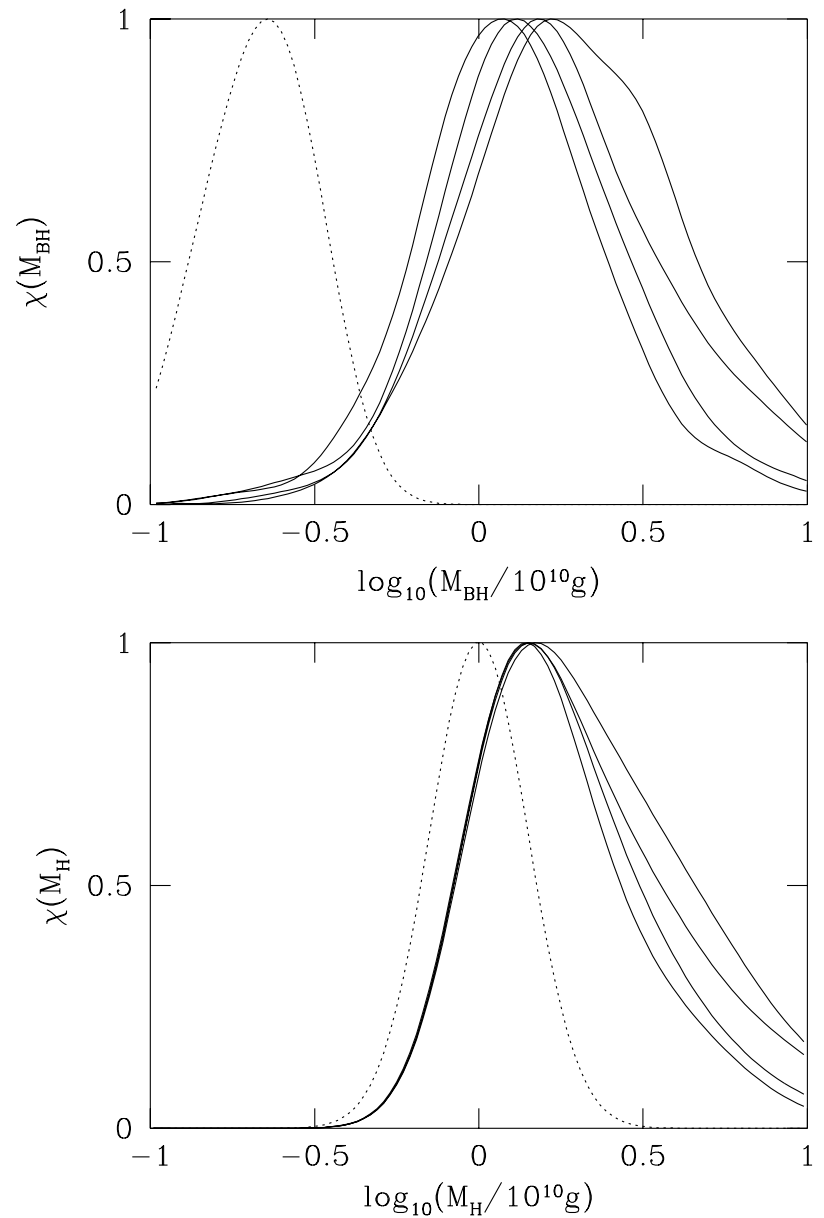

FIG. 2. The smoothed distributions of the actual PBH masses, $\chi\left(M_{\mathrm{BH}}\right)$, (top panel) and the horizon masses at their formation, $\chi\left(M_{\mathrm{H}}\right)$, (lower panel). From top to bottom on the right-hand side of each diagram, the solid lines are for $n=1.310,1.305,1.300$ and 1.295 . In the top panel the dotted line shows the Niemeyer and Jedamzik mass function, evaluated for $\sigma\left(10^{10} \mathrm{~g}\right)=0.032$ and smoothed on the same scale, while in the bottom panel the dotted line is a smoothed delta function, centered on $M_{\mathrm{H}}=10^{10} \mathrm{~g}$.

\section{SPIKY POWER SPECTRA}

Observations of microlensing of stars in the Large Magellanic Cloud [15] appear to show that a large fraction of the halo of the galaxy is in the form of MAssive Compact Halo Objects (MACHOs) with masses of $M \sim 0.5 M_{\odot}$. It has been proposed that MACHOs may be PBHs formed either during the QCD epoch [16,17] because of the reduction in pressure forces at that time, or due to a spike in the primordial density perturbation spectrum [18] at the scale corresponding to $M_{\mathrm{H}} \sim 10^{33} \mathrm{~g}$.

The mass distribution of the MACHOs is steeply peaked. In Ref. [15] the observed microlensing events are fitted with power-law mass functions:

$$
\psi(M)=A M^{\alpha}\left(M_{\min }<M<M_{\max }\right)
$$

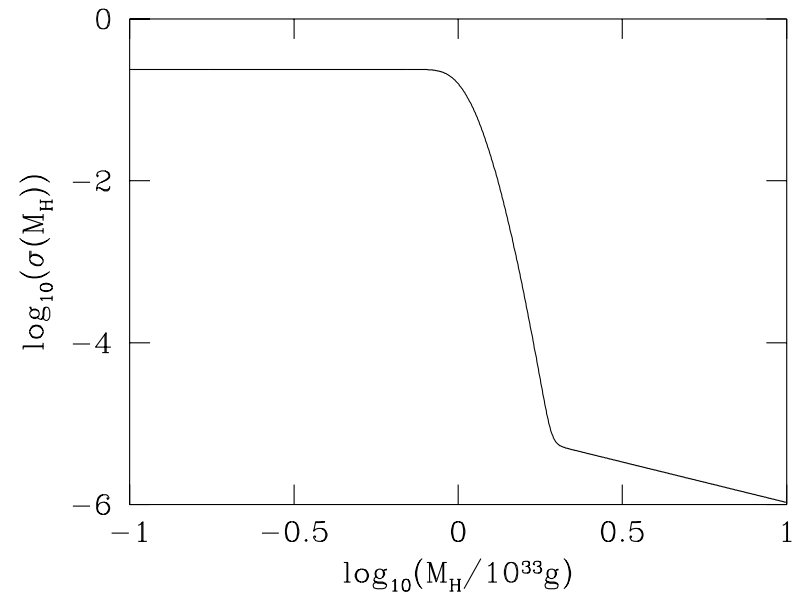

FIG. 3. The form of $\sigma\left(M_{\mathrm{H}}\right)$ produced by a spike in the density perturbation spectrum with $C=1 \times 10^{10} \mathrm{Mpc}^{-1}$ and $\Sigma=2 \times 10^{9} \mathrm{Mpc}^{-1}$.

$$
=0 \text { (otherwise), }
$$

where $\psi(M) \mathrm{d} M$ is the mass fraction of MACHOs between $M$ and $M+\mathrm{d} M$ and $\mathrm{A}$ is determined by the mass fraction of the halo in the form of MACHOs. The maximum likelihood fit (for their 8 event sample) is found for $\alpha=-3.9$ and $M_{\min }=0.30 M_{\odot} \cdot \beta$

The spread in the masses of PBHs formed at a single horizon mass, found when critical collapse is taken into account, raises the question as to whether or not it is possible to produce a population of $\mathrm{PBHs}$ with a sufficiently sharply peaked mass distribution. There are several models of inflation which produce a spike in the power spectrum on a particular scale [18]. For generality, we will take the spectrum of the curvature perturbations, $\mathcal{P}_{\mathcal{R}}$, which is defined, analogously to $\mathcal{P}_{\delta}$, as $[10$

$$
\mathcal{P}_{\mathcal{R}}=\frac{k^{3}}{2 \pi^{2}}\langle|\mathcal{R}(\mathbf{k})|\rangle,
$$

to have the form of a gaussian spike at a scale $k_{\mathrm{c}}=$ $k_{\text {eq }}\left(M_{\mathrm{H}, \text { eq }} / M_{\mathrm{H}, \mathrm{c}}\right)^{0.5}=4.25 \times 10^{10} \mathrm{Mpc}^{-1}$, corresponding to $M_{\mathrm{H}, \mathrm{c}}=10^{33} \mathrm{~g}$, with variable amplitude and width, $\Sigma$, superimposed on a flat spectrum normalized to the COBE data on the present horizon scale. The COBE normalization gives $\mathcal{P}_{\mathcal{R}}=2.28 \times 10^{-9}$ [10 so our spectrum is

$$
\mathcal{P}_{\mathcal{R}}=2.28 \times 10^{-9}+\frac{C}{\sqrt{2 \pi} \Sigma} \exp \left(-\frac{\left(k-k_{\mathrm{c}}\right)^{2}}{2 \Sigma^{2}}\right),
$$

In practice the results from taking a spectrum of this form are quite general, as the bulk of the $\mathrm{PBH}$ production

\footnotetext{
$\S$ For a subset of 6 events (chosen to exclude a binary lensing event whilst maintaining the mean duration) the most likely mass distribution is a delta function.
} 


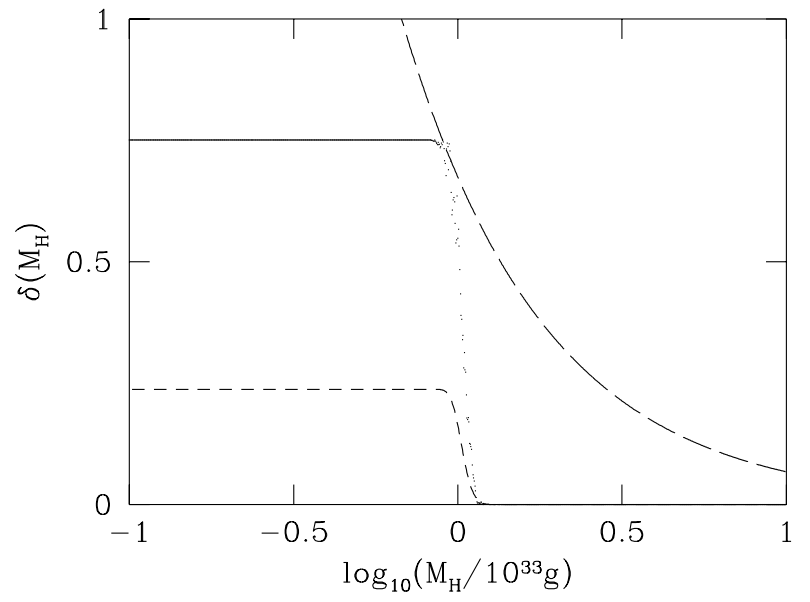

FIG. 4. A PBH forming trajectory produced when $\mathcal{P}_{\mathcal{R}}$ has a gaussian spike with $\Sigma=10^{9} \mathrm{Mpc}^{-1}$ and $C=10^{10} \mathrm{Mpc}^{-1}$. The amplitude jumps dramatically when the spike is encountered. The dotted points are the values of $\delta\left(M_{\mathrm{H}}\right)$, the long-dashed line shows the threshold $\delta_{\mathrm{c}, \mathrm{ft}}$ for $\mathrm{PBH}$ formation, and the short-dashed line shows the typical size of perturbations on each scale $\sigma\left(M_{\mathrm{H}}\right)$.

occurs right at the peak of the power spectrum. A Taylor expansion in the vicinity of the peak requires only the amplitude and curvature, and the two parameters of the gaussian can reproduce an arbitrary function near the peak. Our results will therefore prove extremely general.

With this more complicated spectrum, in order to obtain $\Lambda(M) \equiv \sigma^{2}(M)$ we have to numerically integrate Eq. (位) for each $\mathcal{P}_{\mathcal{R}}$ we choose, where during radiation domination

$$
\delta(\mathbf{k})=\frac{4}{9}\left(\frac{k}{a H}\right)^{2} \mathcal{R}(\mathbf{k})
$$

so that

$$
\mathcal{P}_{\delta}=\frac{16}{81}\left(\frac{k}{a H}\right)^{4} \mathcal{P}_{\mathcal{R}}
$$

Fig. 3 shows $\sigma\left(M_{\mathrm{H}}\right)$ when $C=1 \times 10^{10} \mathrm{Mpc}^{-1}$ and $\Sigma=$ $2 \times 10^{9} \mathrm{Mpc}^{-1}$.

We have run simulations for a range of values of $C$ and $\Sigma$ producing, in each case, $1000 \mathrm{PBHs}$, choosing the fixed time at which the trajectories are produced as the epoch when $M_{\mathrm{H}}=10^{33} \mathrm{~g}$, corresponding to half a solar mass. Density perturbation spectra with the same value of $C$ but different $\Sigma$ have the same value of $\sigma\left(M_{\mathrm{H}}\right)$ on small scales $\left(M_{\mathrm{H}}<10^{33} \mathrm{~g}\right)$, decreasing as $C$ is increased. Once again it is not feasible to use spectra which produce PBH abundances consistent with the observational limits. We therefore choose values of $C$ and $\Sigma$ which lead to larger values of $\sigma\left(M_{\mathrm{H}}\right)$ on small scales $(\sim 0.2)$ and examine the behaviour of the $\mathrm{PBH}$ mass and horizon mass distributions at formation as $C$ and $\Sigma$ are varied. Fig. 4 shows an example of a PBH forming trajectory with $\Sigma=10^{9} \mathrm{Mpc}^{-1}$ and $C=10^{10} \mathrm{Mpc}^{-1}$.
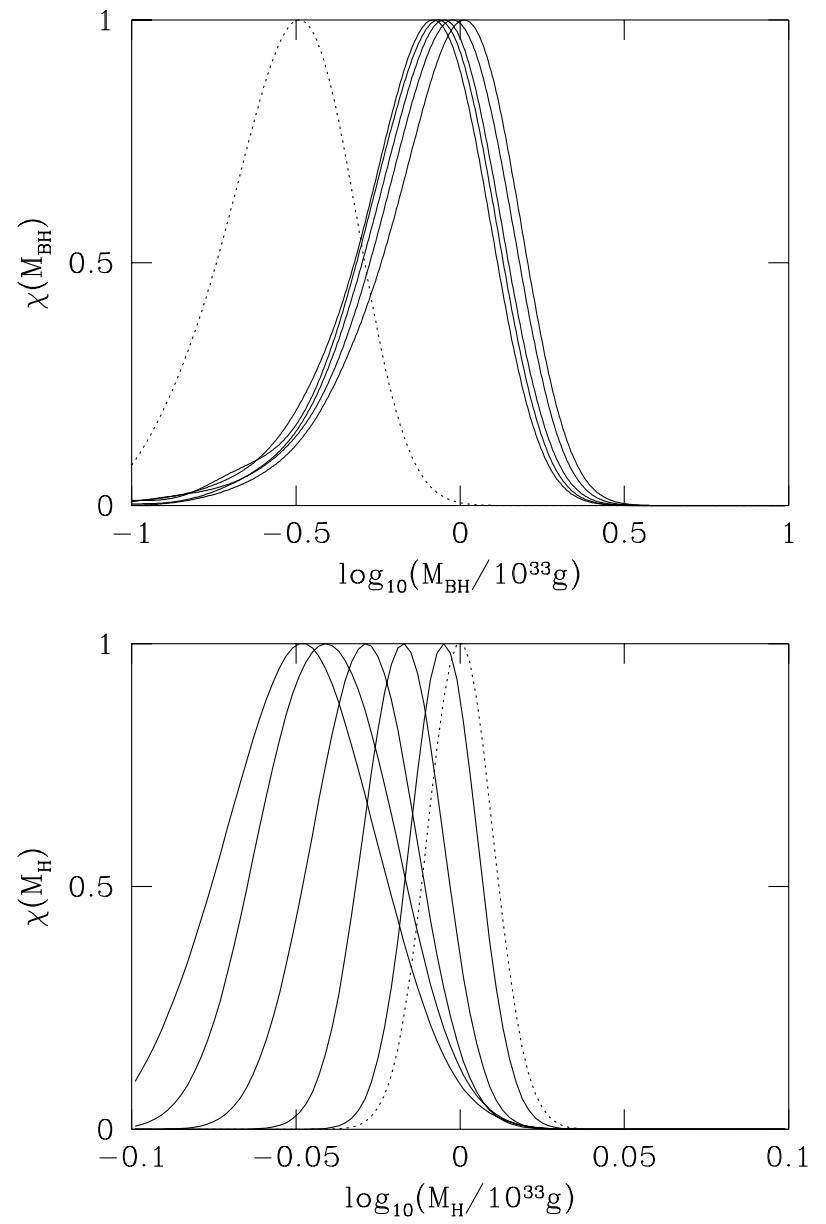

FIG. 5. The smoothed distribution of the actual masses, $\chi\left(M_{\mathrm{BH}}\right)$, (upper panel) and horizon masses, $\chi\left(M_{\mathrm{H}}\right)$, (lower panel) of PBHs formed due to a spike in the density perturbation spectrum with $C=1 \times 10^{10} \mathrm{Mpc}^{-1}$. From right to left on the right-hand side of each diagram, the solid lines show $\Sigma=1 \times 10^{8}, 5 \times 10^{8}, 1 \times 10^{9}, 1.5 \times 10^{9}$ and $2 \times 10^{9} \mathrm{Mpc}^{-1}$. The dotted line in the upper panel shows the smoothed Niemeyer and Jedamzik mass function evaluated for $\sigma\left(10^{33} \mathrm{~g}\right)=0.053$, and in the lower panel a smoothed delta function centered on $M_{\mathrm{H}}=10^{33} \mathrm{~g}$.

The upper panel of Fig. 5 shows the smoothed distribution of the PBH masses, $\chi\left(M_{\mathrm{BH}}\right)$, for $C=1 \times 10^{10} \mathrm{Mpc}^{-1}$ and a range of values of $\Sigma$. For comparison we also plot the smoothed NJMF evaluated for $\sigma\left(M_{\mathrm{H}}=10^{33} \mathrm{~g}\right)=$ 0.053 , the largest value compatible with the requirement that the present-day density of PBHs is consistent with the present-day age and expansion rate of the universe, i.e. $\Omega_{\mathrm{PBH}, 0}<1$. For all the values of $\Sigma$ we used, the PBH mass function has the same shape as the NJMF, but it is centered on larger values of $M_{\mathrm{BH}}$, shifting towards smaller values of $M_{\mathrm{BH}}$ as $\Sigma$ is increased. The lower panel shows the smoothed distribution of the horizon masses at which the PBHs form, $\chi\left(M_{\mathrm{H}}\right)$, for the same set of $C$ and $\Sigma$ values, along with a smoothed delta function centered on $M_{\mathrm{H}}=1 \times 10^{33} \mathrm{~g}$. The horizon mass distributions 

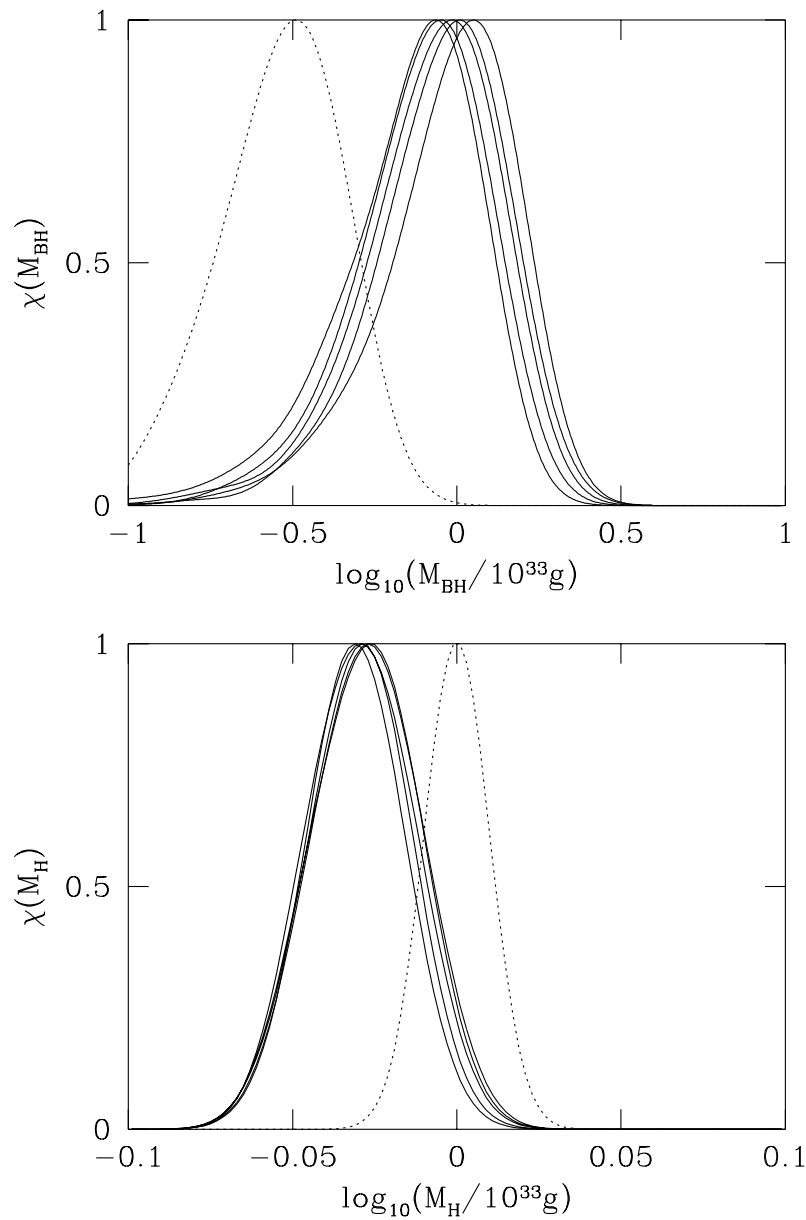

FIG. 6. The smoothed distributions of the actual masses, $\chi\left(M_{\mathrm{BH}}\right)$, (upper panel) and horizon masses, $\chi\left(M_{\mathrm{H}}\right)$ (lower panel) of PBHs formed due to a spike in the density perturbation spectrum with $\Sigma=1 \times 10^{9} \mathrm{Mpc}^{-1}$ and (from right to left on the right-hand side of each diagram) $C=1.75 \times 10^{10}, 1.5 \times 10^{10}, 1.25 \times 10^{10}, 1 \times 10^{10}$ and $7.5 \times 10^{9} \mathrm{Mpc}^{-1}$. The dotted line in the upper panel shows the Niemeyer and Jedamzik mass function evaluated for $\sigma\left(10^{33} \mathrm{~g}\right)=0.053$, and in the lower panel shows a smoothed delta function centered on $M_{\mathrm{H}}=10^{33} \mathrm{~g}$.

have the same shape as the delta function but are centered at smaller $M_{\mathrm{H}}$. As $\Sigma$ is decreased the centre of the distribution tends towards $M_{\mathrm{H}}=10^{33} \mathrm{~g}$.

Fig. 6 shows the same distributions for $\Sigma=1 \times$ $10^{9} \mathrm{Mpc}^{-1}$ and a range of $C$ values. As $C$ is decreased the centre of the PBH mass function tends to that of the NJMF. The distribution of the horizon masses at which the PBHs form is independent of $C$ and centered at $M_{\mathrm{H}}<10^{33} \mathrm{~g}$.

The horizon masses at which the $\mathrm{PBH}$ form are typically smaller than $10^{33} \mathrm{~g}$, since whilst $\mathcal{P}_{\mathcal{R}}$ has a spike centered at this scale, $\sigma\left(M_{\mathrm{H}}\right)$ carries on increasing, as $M_{\mathrm{H}}$ is decreased, until $\mathcal{P}_{\mathcal{R}} \ll C$ on that scale. By increasing the scale on which the spike is centered we could tune the horizon mass distribution to be precisely centered at

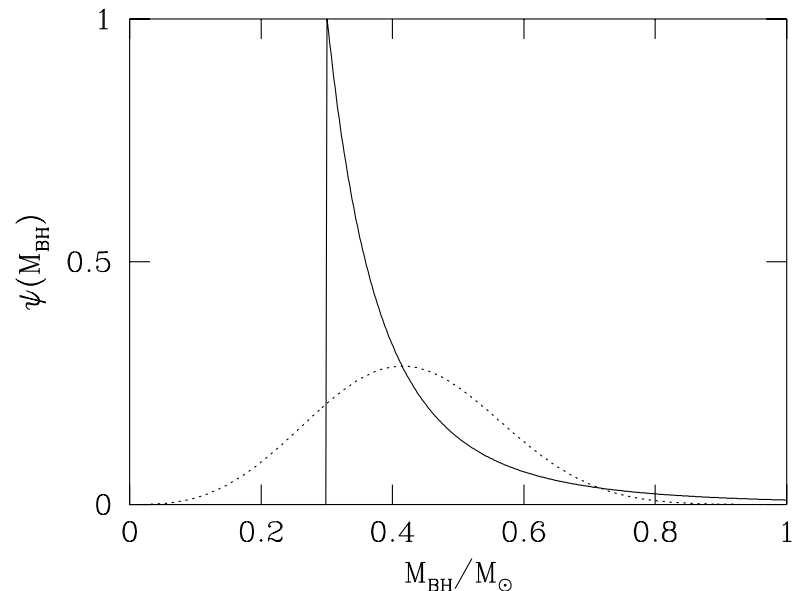

FIG. 7. The observed distribution of the MACHOs, given by Eq. (19), is shown as the solid line and the Niemeyer and Jedamzik mass function, given by Eq. (18), as the dashed line. We fix $M_{\mathrm{H}}=2.05 \times 10^{33} \mathrm{~g}$ so that the mass functions have the same mean. The curves are chosen to have the same integrated mass fraction and the same mean.

$M_{\mathrm{H}}=10^{33} \mathrm{~g}$.

The PBH masses are typically larger than given by the NJMF with $\sigma\left(10^{33}\right) \mathrm{g}=0.053$, despite being formed at smaller $M_{\mathrm{H}}$. This is because our spiky density perturbation spectra all have $\sigma\left(M_{\mathrm{H}}\right) \sim 0.2$, in order to produce a larger number of $\mathrm{PBH}$ s than is allowed by observations (as is discussed above), leading to larger values of $\delta(M)$ and hence $M_{\mathrm{BH}}$.

Since the spread of $M_{\mathrm{H}}$ at which the PBHs form will be extremely small if the amplitude of the spike, and hence $\sigma(M)$, is reduced so that the number of $\mathrm{PBH}$ s produced satisfies the constraint $\Omega_{(\mathrm{PBH}, 0)}<1$, then the $\mathrm{PBH}$ mass function will be the same, to a good approximation, as that found by assuming that all the PBHs form at a single horizon mass. We therefore compare, in Fig. 7, the observed MACHO mass function, $\psi(M)$, with that determined by Niemeyer and Jedamzik, Eq. (18). Defining $\tilde{\psi}(M) \mathrm{d}(\ln M)$ to be the mass fraction in a logarithmic mass interval $\mathrm{d}(\ln M)$, then $\tilde{\psi}(M)=\psi(M) M$. Since $\mathrm{d} N / \mathrm{d}(\ln M) \propto \tilde{\psi} / M$, then $\psi\left(M_{\mathrm{BH}}\right) \propto \mathrm{d} N / \mathrm{d}\left(\ln M_{\mathrm{BH}}\right)$. That is to say, the mass fraction per unit mass interval is just proportional to the number density per logarithmic mass interval. We adjust the horizon mass at which the $\mathrm{PBH}$ s are assumed to form at to $M_{\mathrm{H}}=2.05 \times 10^{33} \mathrm{~g}$, so that the $\mathrm{PBH}$ mass distribution has the same mean mass as the MACHO distribution. The mass distributions are normalized to give the same total mass fraction when integrated.

The PBH mass distribution is much broader than that fitted to the observed microlensing events; its full width at half maximum is six times larger (assuming the mass functions are normalized to the same mean). Because the derivation assumes all black holes form at the same horizon mass, it is the narrowest possible mass func- 
tion which can arise if the critical collapse hypothesis is correct. The mass function is almost symmetrical, extending to $M_{\mathrm{BH}} \ll M_{\mathrm{H}}$. Currently there is a robust limit, due to the absence of short-duration microlensing events, that less than 20 percent of the dark matter in the halo of the galaxy can be in low mass MACHOs $\left(10^{-4} M_{\odot}<M<0.03 M_{\odot}\right)$ [15], but the PBH mass function is easily consistent with that. Whether or not the width of the predicted mass function is in agreement with the observed microlensing events, which so far have been fitted with much narrower functions, is a much more pertinent question. As the duration of microlensing searches increases, and further microlensing events are observed, the MACHO mass function will be determined much more accurately, and if the MACHOs are $\mathrm{PBHs}$ then the true MACHO mass function will be considerable wider than the sharply-peaked mass functions which have been fitted to the current data.

\section{CONCLUSIONS}

Critical gravitational collapse, and in particular the scaling relation for the black hole mass, has an astrophysical application to PBH formation. We have used the excursion set formalism to determine the PBH mass function, when formation on a range of mass scales is taken account of, for two different types of power spectra. The first is power-law density perturbation spectra, and the second is flat spectra with a spike on a given scale. In both cases we find that as the parameters are adjusted so that the abundance of PBHs decreases, the mass function tends towards that found by Niemeyer and Jedamzik under the assumption that all the PBHs form at a single horizon mass. There are tight observational limits on the abundance of PBHs, and for power spectra which satisfy these constraints the assumption that all $\mathrm{PBHs}$ form at the same horizon mass is a good approximation.

As an application, we then compared the Niemeyer and Jedamzik PBH mass function with that of the MACHOs found from microlensing observations. The $\mathrm{PBH}$ mass distribution is considerably broader than the sharply-peaked simple mass distributions which have so far been fitted to the observed microlensing events. If the MACHOs are indeed PBHs, then future microlensing searches should unveil the broad spread of the PBH mass distribution.

\section{ACKNOWLEDGMENTS}

A.M.G. was supported by PPARC and A.R.L. in part by the Royal Society. We thank Bernard Carr, Karsten Jedamzik and Jens Niemeyer for useful discussions. A.M.G. acknowledges use of the Starlink computer system at QMW.
[1] C. Gundlach, Adv. Theor. Math. Phys. 2, 1 (1998).

[2] M. W. Choptuik, Phys. Rev. Lett. 70, 9 (1993).

[3] C. R. Evans and J. S. Coleman, Phys. Rev. Lett. 72, 1782 (1994).

[4] J. C. Niemeyer and K. Jedamzik, Phys. Rev. Lett. 80, 5481 (1998).

[5] J. C. Niemeyer and K. Jedamzik, preprint astro$\mathrm{ph} / 9901292$.

[6] B. J. Carr, Astrophys. J. 201, 1 (1975).

[7] B. J. Carr and S. W. Hawking, Mon. Not. R. Astron. Soc. 168, 399 (1974).

[8] J. R. Bond, S. Cole, G. Efstathiou and N. Kaiser, Astrophys. J. 379, 440 (1991); C. Lacey and S. Cole, Mon. Not. R. Astron. Soc. 262, 627 (1993); C. Lacey and S. Cole, Mon. Not. R. Astron. Soc. 271, 676 (1994).

[9] C. Porciani, S. Matarrese, F. Lucchin and P. Catelan, Mon. Not. R. Astron. Soc. 298, 1097 (1998).

[10] A. R. Liddle and D. H. Lyth, Phys. Rep. 231, 1 (1993).

[11] J. S. Bullock and J. R. Primack, Phys. Rev. D 55, 7423 (1997); P. Ivanov, Phys. Rev. D 57, 7145 (1998).

[12] A. M. Green and A. R. Liddle, Phys. Rev. D 54, 6166 (1997).

[13] B. J. Carr, in Current Topics in Astrofundamental Physics, proceedings of the International School of Astrophysics 'D. Chalonge' 1996, edited by N. Sanchez and A. Zichichi (World Scientific Singapore).

[14] J. Yokoyama, Phys. Rev. D. 58, 107502 (1998).

[15] C. Alcock et al., Astrophys. J. 486, 697 (1997).

[16] M. Crawford and D. N. Schramm, Nature 298, 538 (1982).

[17] K. Jedamzik, Phys. Rev. D 55, R5871 (1997); D. J. Schwarz, C. Schmid and P. Widerin, Phys. Rev. Lett. 78, 791, (1997); C. Schmid, D. J. Schwarz and P. Widerin, Phys. Rev. D 59, 043517 (1999).

[18] P. Ivanov, P. Naselsky and I. Novikov, Phys. Rev. D 50, 7173 (1994); J. Yokoyama, astro-ph/9509027; L. Randall, M. Soljačić and A. H. Guth, Nucl. Phys. B. 472, 377 (1996); J. García-Bellido, A. Linde and D. Wands, Phys. Rev. D 54, 6040 (1996). 